\title{
Regulation of Mitochondrial Transport and Inter-Microtubule Spacing by Tau Phosphorylation at the Sites Hyperphosphorylated in Alzheimer's Disease
}

\author{
Kourosh Shahpasand, ${ }^{1}$ Isao Uemura, ${ }^{2}$ Taro Saito, ${ }^{1}$ Tsunaki Asano, ${ }^{3}$ Kenji Hata, ${ }^{4}$ Keitaro Shibata, ${ }^{5}$ Yoko Toyoshima, ${ }^{5}$ \\ Masato Hasegawa, ${ }^{6}$ and Shin-ichi Hisanaga ${ }^{1}$ \\ ${ }^{1}$ Laboratory of Molecular Neuroscience, ${ }^{2}$ Laboratory of Developmental Program, ${ }^{3}$ Laboratory of Cell Genetics, ${ }^{4}$ Laboratory of Plant Ecology, Department of \\ Biological Sciences, Tokyo Metropolitan University, Minami-osawa, Hachioji, Tokyo 192-0397, Japan, ${ }^{5}$ Department of Life Sciences, Graduate School of \\ Arts and Sciences, The University of Tokyo, Komaba, Tokyo 153-8902, Japan, and ${ }^{6}$ Tokyo Metropolitan Institute of Medical Sciences, Department of \\ Neuropathology and Cell Biology, Setagaya-Ku, Tokyo 156-0057, Japan
}

The microtubule-associated protein Tau is a major component of the neurofibrillary tangles that serve as a neuropathological hallmark of Alzheimer's disease. Tau is a substrate for protein phosphorylation at multiple sites and occurs in tangles in a hyperphosphorylated state. However, the physiological functions of Tau phosphorylation or how it may contribute mechanistically to Alzheimer's pathophysiology are not completely understood. Here, we examined the function of human Tau phosphorylation at three sites, Ser199, Ser202, and Thr205, which together comprise the AT8 sites that mark abnormal phosphorylation in Alzheimer's disease. Overexpression of wild-type Tau or mutated forms in which these sites had been changed to either unphosphorylatable alanines or phosphomimetic aspartates inhibited mitochondrial movement in the neurite processes of PC12 cells as well as the axons of mouse brain cortical neurons. However, the greatest effects on mitochondrial translocation were induced by phosphomimetic mutations. These mutations also caused expansion of the space between microtubules in cultured cells when membrane tension was reduced by disrupting actin filaments. Thus, Tau phosphorylation at the AT8 sites may have meaningful effects on mitochondrial movement, likely by controlling microtubule spacing. Hyperphosphorylation of the AT8 sites may contribute to axonal degeneration by disrupting mitochondrial transport in Alzheimer's disease.

\section{Introduction}

Mitochondrial transport within axons is crucial for axonal maintenance, and its dysregulation can contribute to neurodegenerative diseases (Su et al., 2010). In axons, mitochondrial movement is driven by two oppositely directed motor proteins, kinesin and dynein, along microtubules (MTs) (Hollenbeck and Saxton, 2005; Bereiter-Hahn and Jendrach, 2010). The surface of MTs is decorated with microtubule-associated proteins (MAPs) (Marx et al., 2006; Vershinin et al., 2007). Tau serves as a predominant MAP in axons and is a filamentous protein of 441 amino acid residues (the longest human isoform). Tau is comprised of two functional regions, the $\mathrm{N}$-terminal projection domain that pro-

Received Nov. 29, 2011; accepted Dec. 12, 2011.

Author contributions: K. Shahpasand and S.-i.H. designed research; K. Shahpasand performed research; I.U., T.S., T.A., K. Shibata, Y.T., and M.H. contributed unpublished reagents/analytic tools; K. Shahpasand, I.U., T.S., K.H., and S.-i.H. analyzed data; K. Shahpasand and S.-i.H. wrote the paper.

This work was supported by Grants-in-Aid for Scientific Research on Priority Area from MEXT of Japan (S.H). K. S. was supported by JGC-SScholarship Foundation. We thank Dr. Peter Davies at Albert Einstein College of Medicine for providing MC- 1 and Alz-50 monoclonal anti-Tau antibodies. We also thank Miss. Elizabeth Zielinska for reading this paper.

Correspondence should be addressed to either Kourosh Shahpasand or Shin-ichi Hisanaga. Laboratory of Molecular Neuroscience, Department of Biological Sciences, Tokyo Metropolitan University, Minami-osawa 1-1, Hachioji, Tokyo 192-0391, Japan. E-mail: kourosh-shahpasand@ed.tmu.ac.jp or hisanaga-shinichi@tmu.ac.jp.

DOI:10.1523/JNEUROSCI.5927-11.2012

Copyright $\odot 2012$ the authors $\quad 0270-6474 / 12 / 322430-12 \$ 15.00 / 0$ trudes from the surface of MTs and the C-terminal MT-binding domain. Overexpression of Tau inhibits mitochondrial transport in various cells (Ebneth et al., 1998; Trinczek et al., 1999; Stamer et al., 2002; Dixit et al., 2008; Stoothoff et al., 2009; Vossel et al., 2010). However, the mechanism and regulation of Tau-mediated inhibition of mitochondrial transport are not understood.

Tau is a major component of neurofibrillary tangles found in Alzheimer pathology. Tau is also a phosphoprotein, the functions of which can be regulated by phosphorylation (Stoothoff and Johnson, 2005; Hanger et al., 2009). Many of the phosphorylation sites reside in proline-directed (Ser/ Thr)-Pro sequences. These sites are moderately phosphorylated in healthy neurons. However, hyperphosphorylation is linked to neurodegeneration with phosphorylation of more than 20 sites shown in the degenerated brains of Alzheimer's patients (Watanabe et al., 1993; Morishima-Kawashima et al., 1995; Stoothoff and Johnson, 2005). Cdk5 and GSK3 $\beta$ are two prolinedirected protein kinases that are known to phosphorylate these (Ser/Thr)-Pro sites (Ishiguro et al., 1992; Planel et al., 2002). Furthermore, hyperactivation of $\mathrm{Cdk} 5$ or GSK $3 \beta$ reduces mitochondrial movement (Darios et al., 2005; Morel et al., 2010). However, it has not been clearly demonstrated whether Tau is a major downstream target of these kinases and, if so, which phosphorylation site(s) is critical. Among GSK3 $\beta$ - and Cdk5-related phosphorylation sites, Ser199, Ser202, and Thr205 are particu- 
larly interesting. These phosphorylated sites are recognized by the phosphorylation-dependent monoclonal antibody AT8 (Goedert et al., 1995; Wang et al., 2006; Hanger et al., 2009), and their detection by this antibody is commonly used as a marker of abnormal phosphorylation in brains of Alzheimer's patients. These sites are located at the border of the projection domain and MTbinding Pro-rich region (see Fig. $1 A$ ). Phosphorylation at these sites may extend the projection domain outwards from the surface of MTs (Jeganathan et al., 2008) and affect mitochondrial transport (Mukhopadhyay and Hoh, 2001; Jeganathan et al., 2008; Shahpasand et al., 2008).

Here, we directly examined the effect of phosphorylation at the AT8 sites on mitochondrial transport using the nonphosphorylatable Ala mutant (3A) and the constitutive phosphorylation mimic Asp mutant (3D) of Tau. Overexpression of Tau 3D decreased the mitochondrial movement and expanded the inter-MT spacing more than that of wild-type (WT) Tau or Tau 3A. These results suggest that phosphorylation at the AT8 sites affects mitochondrial transport by changing the spaces between MTs.

\section{Materials and Methods}

Anti-human Tau (A0024) was obtained from Dako. Anti-phospho-Tau antibody AT8 was purchased from Thermo Scientific. Anti-TOM20 (FL145) was from Santa Cruz Biotechnology. MC-1 and Alz-50 anti-Tau antibodies were generous gifts from Dr. Peter Davies (Albert Einstein College of Medicine, Bronx, NY). Anti- $\beta$-tubulin and latrunculin B were from Sigma-Aldrich. Anti-His tag was from Invitrogen. NGF and Phostag acrylamide was obtained from Wako Chemicals.

Construction of expression vectors for Tau proteins. Mutations of Tau at Ser199, Ser202, and Thr205 were introduced in the longest wild-type human Tau in a pSG5 expression vector. Double Ala mutants (2A) at Ser202A and Thr205A were constructed with PCR using primers 5'-GGCGCCCCAGG CGCTCCCGGCAGCCGC-3' (forward) and 5' -CCGCGGGGTCCGCGAGGGCCGTCGGCG-3' (reverse). For Tau 3A, an additional Ala mutation at Ser199 was constructed with PCR using Tau 2A as a template and primers 5'-AGCGGCTACAGCGCCCCCGGCGCCCCA-3' (forward) and 5'-TCGCCGATGTCGCGGGGGCCGCGGGGT-3' (reverse). Tau 3D was constructed by one-step PCR using Tau 3A as a template and primers $5^{\prime}$ AGCGGCTACAGCGACCCCGGCGACCCAGGCGATCCCGGCAGCCGC-3' (forward) and 5' -TCGCCGATGTCGCTGGGGCCGCTGGGTCC GCTAGGGCCGTCGGCG-3' (reverse). All constructs were confirmed by DNA sequencing. Bacterial expression vectors encoding Tau 3A and Tau 3D were constructed similarly with PCR using Tau 1N4R (one N-terminal insertion and four MT-binding repeats) in the pRK172 vector. Baculovirus expression vectors for Tau proteins were constructed as follows. Tau WT, $3 \mathrm{~A}$, or 3D in $\mathrm{PSG} 5$ was amplified with PCR using primers 5' -GGATCCATGGCTGAGCCCCGCCA-3' (forward, with BamHI site) and 5'-GCGGCCGCTCACAAACCCTGCTT-3' (reverse, with NotI site). PCR products were inserted into the pCR2.1 vector. Tau cDNAs were digested with BamHI and NotI and inserted into pFastBac Dual, the donor vector for the Bac-toBac Baculovirus Expression System (Invitrogen). Recombinant plasmids were used to transform competent DH10Bac cells for transposition to the bacmid shuttle vectors. Recombinant bacmid DNA was transfected into monolayers of $\mathrm{Sf} 9$ insect cells. Supernatants containing virus were harvested $96 \mathrm{~h}$ after transfection, and the viral titer was amplified and determined according the manufacturer's instructions (Invitrogen).

Cell culture and expression of Tau proteins. COS-7 cells $\left(2 \times 10^{5}\right.$ per ml $)$ were grown in DMEM (Sigma) containing 10\% FBS and transfected with PolyFect reagent (Qiagen) (Kaminosono et al., 2008).

PC12 cells $\left(2 \times 10^{5}\right.$ per $\left.\mathrm{ml}\right)$ were cultured on $35 \mathrm{~mm}$ glass-bottom dishes in DMEM containing 10\% FBS and antibiotics (penicillin and streptomycin) at $37^{\circ} \mathrm{C}$ in $5 \% \mathrm{CO}_{2}$. Cells were treated with NGF (50 $\mathrm{ng} / \mathrm{ml}) 4 \mathrm{~h}$ after transfection for $3 \mathrm{~d}$.

Primary neurons were prepared from 17-day-old embryonic mouse brain cerebral cortex of either sex. Neurons $\left(2 \times 10^{5}\right.$ per ml $)$ were seeded on $35 \mathrm{~mm}$ glass-bottom dishes. The medium was then changed to neu- robasal medium supplemented with B27 (Invitrogen) and $1 \mathrm{~mm}$ L-glutamine (Endo et al., 2009). Cells were transiently transfected with Tau WT, 3A, or 3D with Lipofectamine 2000 (Invitrogen). Various amounts of Tau cDNA (1-4 $\mu \mathrm{g})$ were tested to determine the appropriate amount of cDNA. The levels of Tau expression were quantified by immunostaining intensity as described below. Neurons were cotransfected with Mito-GFP to label and observe mitochondrial movement.

Sf9 cells were grown at $27^{\circ} \mathrm{C}$ in $35 \mathrm{~mm}$ dishes in complete serum-free medium (Invitrogen ) and antibiotics (penicillin and streptomycin). Monolayer cultures of Sf9 cells $\left(4 \times 10^{5}\right.$ per $\left.\mathrm{ml}\right)$ were infected with baculoviruses encoding Tau cDNA at a titer of $2 \times 10^{7} \mathrm{pfu} / \mathrm{ml}$. Cells were treated with $0.5 \mu \mathrm{g} / \mathrm{ml}$ latrunculin B from the time of infection for $72 \mathrm{~h}$ and were fixed with $2.5 \%$ glutaraldehyde and 2\% PFA in PEM buffer (0.1 м PIPES, pH 7.2, 1 mM EGTA, $1 \mathrm{~mm} \mathrm{MgCl}_{2}$ ) for $30 \mathrm{~min}$ at room temperature.

Immunostaining. One day after transfection, COS-7 cells were treated with PEM buffer containing $4 \mathrm{M}$ glycerol and $0.5 \%$ Triton X-100 for 10 $\min$ at $37^{\circ} \mathrm{C}$. After fixation with cold methanol for $3 \mathrm{~min}$, cells were incubated with anti-tubulin (1:500) or anti-Tau (1:500), followed by Alexa Fluor-conjugated anti-mouse IgG or anti-rabbit IgG (1:500).

Sf9 cells, PC1 2 cells, and cortical neurons cultured on glass coverslips were fixed with $4 \%$ PFA in PBS for 20 min and permeabilized with $0.1 \%$ Triton X-100 in PBS containing 5\% BSA for 20 min. Cells were incubated with anti-Tau (1:500) followed by Alexa Fluor 546-labeled secondary antibody. Immunofluorescent stainings were examined with an LSM5 EXCITER microscope (Zeiss), and fluorescent intensities were measured for 20 processes per sample with ZEN 2008 software (Zeiss). Length and width of Sf9 cell processes were measured for 20 cells per sample with ImageJ software.

Electron microscopy. Sf9 cells were infected with baculovirus encoding Tau constructs and $72 \mathrm{~h}$ after infection cells were fixed and processed for electron microscopic observation (Tokuoka et al., 2000). PC12 cells were transfected with plasmids encoding Tau and EGFP. Tau-expressing PC12 cells were identified with EGFP after fixation, and their positions were marked on the base of culture dishes by a felt pen. Fixation was done with $2.5 \%$ glutaraldehyde and 2\% PFA in PEM buffer (0.1 м PIPES, $\mathrm{pH}$ 7.2, $1 \mathrm{~mm}$ EGTA, $1 \mathrm{~mm} \mathrm{MgCl}_{2}$ ) for $30 \mathrm{~min}$ at room temperature. Specimens were examined with a JEM-1010 transmission electron microscope (JEOL). Inter-MT distances in sections cut perpendicularly to longitudinal axis of MTs were measured with ImageJ software.

Time-lapse imaging and statistical methods. Mitochondrial movement in NGF-treated PC12 cells and mouse brain cortical neurons was examined with an LSM 5 EXCITER microscope using an incubation chamber with $5 \% \mathrm{CO}_{2}$ at $37^{\circ} \mathrm{C}$. Fluorescent images of mitochondria in the longest process of each PC12 cell and axons of cortical neurons were acquired at intervals of $5 \mathrm{~s}$ over a period of $300 \mathrm{~s}$. In each experiment, eight mitochondria in five cells were analyzed for their movement in image stacks composed of 60 images. Individual mitochondrial movements were analyzed with ZEN 2008 software (Zeiss). Differences in the position of each mitochondrion between two frames during each $5 \mathrm{~s}$ interval were exported to Excel, and they were classified and scored as stationary, anterograde, or retrograde movements. Completely immotile mitochondria during $300 \mathrm{~s}$ of observation were excluded from the counts. Kymographs were made using serial frames of the same area during the observation period.

Statistical differences in stationary phase of mitochondria among control, Tau WT, 3A, and 3D were analyzed by one-way ANOVA. Holm's multiple test was conducted on all possible pairwise combinations. Differences in the ratios between anterograde and retrograde movements were analyzed by ANCOVA. The dependent variable was time of anterograde movement, and the independent variables were Tau transfection samples as a fixed factor and time of retrograde movement as a covariate. After confirming no interaction between Tau transfection samples and the retrograde ratios, the anterograde/retrograde moving durations were compared using all data or data of Tau-transfected samples. When $p>0.05$, the difference was considered nonsignificant. All of the statistical analyses were carried out with version 2.13.1 of the R software package ( $\mathrm{R}$ : A language and environment for statistical computing. R Development Core Team, Vienna, Austria, 2011, available at http://www.R-project.org). 
Protein purification, motor-MT binding assay, and in vitro phosphorylation of Tau by Cdk5-p25. Tau WT, 3A, or 3D was expressed in Escherichia coli and purified (Sakaue et al., 2005). Porcine brain tubulin was also purified as described (Sakaue et al., 2005). Kinesin RK430-AviTag-KRC-His ${ }_{6}$ was expressed and purified from E. coli as described (Furuta et al., 2008), except that ATP was not added to the preparation buffers. Tubulin $(0.5 \mu \mathrm{M})$ was assembled in PEM containing $1 \mathrm{mM}$ GTP and 10 $\mu \mathrm{M}$ Taxol in the presence of various concentrations of Tau WT, 3A, or 3D $(0.05,0.1$, or 0.15 $\mu \mathrm{M})$ and $0.1 \mu \mathrm{M}$ RK430 at $37^{\circ} \mathrm{C}$ for $45 \mathrm{~min}$. Polymerized MTs were collected by centrifugation at $100,000 \times g$ for $60 \mathrm{~min}$ at $37^{\circ} \mathrm{C}$. The supernatants and pellets were subjected to SDS-PAGE followed by immunoblotting. Cdk5-p25 was purified from Sf9 cells that had been infected with baculovirus encoding Cdk5 and p 25 (Saito et al., 2003). Phosphorylation of Tau by Cdk5-p25 was performed in vitro by incubating $50 \mu \mathrm{g} / \mathrm{ml}$ Tau WT, 3D, or 3A with Cdk5-p25 at $37^{\circ} \mathrm{C}$ for $2 \mathrm{~h}$.

SDS-PAGE and immunoblotting. SDS-PAGE and immunoblotting were performed as described (Sakaue et al., 2005). Phos-tag SDSPAGE was performed with 7.5\% polyacrylamide and $50 \mu \mathrm{M}$ Phos-tag as described (Hosokawa et al., 2010). Dot blot was performed with MC-1 and Alz-50 antibodies using the method reported previously (Jicha et al., 1997). All experiments were performed at least three times, and representative results are shown.

\section{Results}

\section{Phosphorylation of Tau mutants and} their binding to MTs

Tau can be phosphorylated at multiple sites in vitro and in vivo. To assess the effects of site-specific phosphorylation on mitochondrial transport, we used the Tau mutants $3 \mathrm{~A}$ (nonphosphorylatable) and 3D (phosphorylation mimic), each of which was mutated at the AT8 Alzheimer phosphorylation sites (Ser199, Ser202, and Thr205) (Fig. 1A). Recombinant Tau WT, 3A, and $3 \mathrm{D}$ were phosphorylated in vitro by Cdk5-p25 and immunoblotted with AT8, which recognizes Tau that is phosphorylated at Ser202 and Thr205 (Goedert et al., 1995) or Ser199, Ser202, and Thr205 (Hanger et al., 2009). Phosphorylated Tau WT was immunoreactive to AT8, whereas phosphorylated Tau 3A and 3D were not (Fig. 1 B), confirming that Cdk5 indeed phosphorylated the sites recognized by AT8 (Takahashi et al., 2003) and that these phosphorylation sites were disrupted in $3 \mathrm{~A}$ and $3 \mathrm{D}$ constructs. These Tau constructs were expressed in COS-7 cells, and phosphorylation was examined by immunoblotting with AT8. Immunodetection of Tau WT but not 3A nor 3D clearly demonstrated that Tau WT was phosphorylated at the AT8 recognition sites in COS-7 cells (Fig. $1 C$, middle).

To determine whether the mutations affected phosphorylation of Tau at other sites, Tau expressed in COS-7 cells was detected by immunoblotting with a phosphorylation-independent Tau antibody after Phos-tag SDS-PAGE. Phos-tag SDS-PAGE is a recently developed method that detects phosphorylated proteins via an expanded mobility shift on SDS-PAGE (Kinoshita et al., 2006; Hosokawa et al., 2010). Multiple Tau bands indicated the presence of various phosphorylated forms of Tau in COS-7 cells (Fig. 1C, bottom). As a whole, the decreased mobility of Tau WT compared to Tau 3A indicated the higher level of phosphorylation of Tau WT, including the AT8 site. The relative upward shift of all the Tau 3D bands was apparently due to the increased negative charge of the three Asp residues at positions 199, 202, and 205. Phosphorylated Tau 3A and 3D had similar, but not identical, banding patterns, suggesting that these mutants underwent similar phosphorylation at other sites (Fig. $1 D$ ). These results indicated that by using Tau $3 \mathrm{~A}$ and $3 \mathrm{D}$, we could examine the role of Tau phosphorylation at the AT8 sites (Ser199/Ser202/ Thr205) on mitochondrial transport.

To see whether the $3 \mathrm{D}$ mutant mimics the phosphorylation state at the AT8 sites, we performed dot blot of Tau constructs with MC-1 and Alz-50 antibodies after phosphorylation with Cdk5-p25. MC-1 and Alz-50 are monoclonal antibodies that recognize conformation of Tau when phosphorylated at both AT8 and PHF-1 (Ser396 and Ser 404) sites (Jicha et al., 1999). Cdk5phosphorylated Tau WT and Tau 3D, but not Tau 3A, were immunoreactive to $\mathrm{MC}-1$ and Alz-50 (Fig. $1 E$ ). The results indicated that Tau 3D in fact mimics phosphorylation at the AT8 sites.

To assess Tau 3A and 3D binding to MTs, COS-7 cells were transfected with vectors that mediated expression of WT, 3A, or 3D forms of Tau. The Tau-expressing cells were then incubated in a MT-stabilizing buffer containing $0.5 \%$ Triton X-100 and $4 \mathrm{M}$ glycerol to remove soluble tubulin and Tau and then stained with anti-tubulin and anti-Tau (Bershadsky et al., 1978; Kaminosono 
Tau
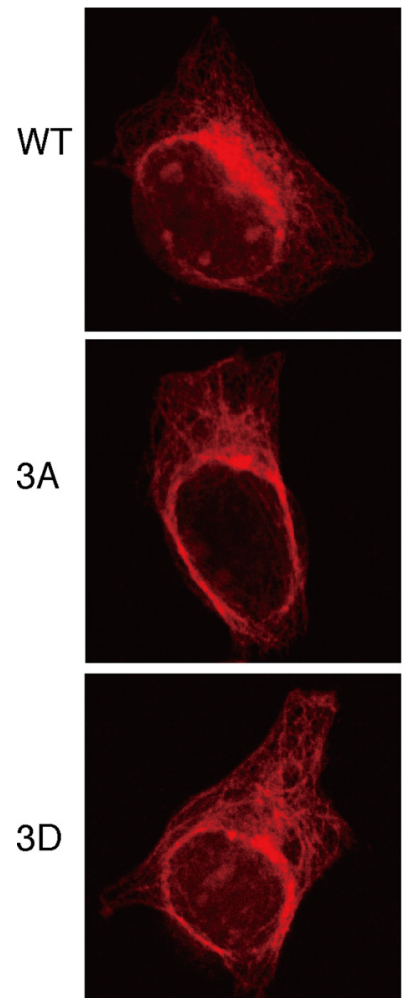

Tubulin
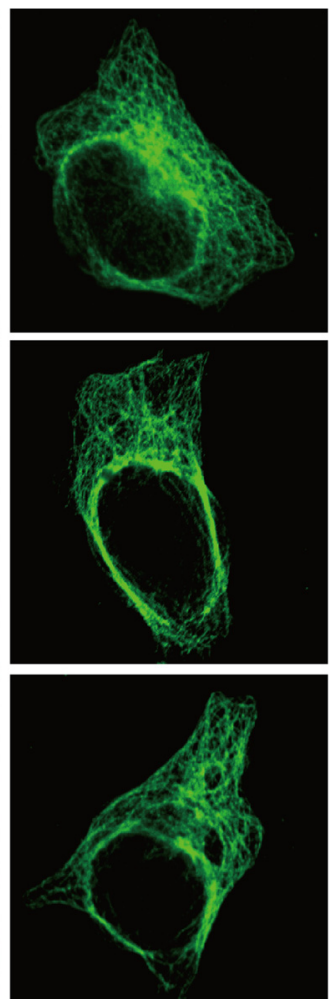

Merge
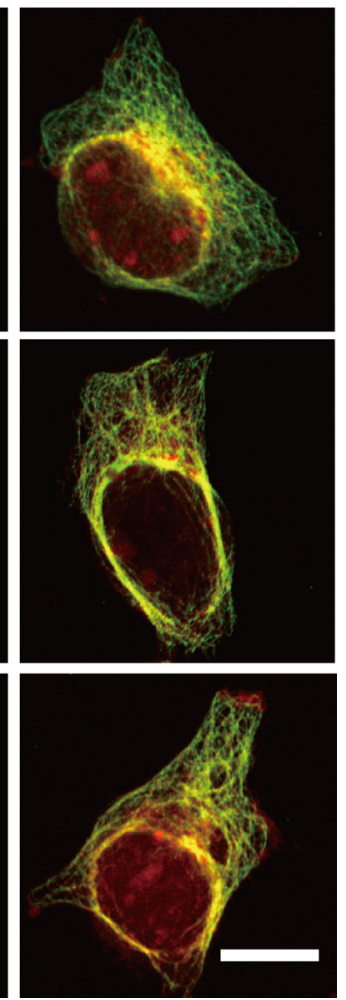

Figure 2. Colocalization of Tau WT, 3A, or 3D to MTs in COS-7 cells. Tau WT, 3A, or 3D vectors were used to transfect COS-7 cells. Soluble Tau and tubulin were removed by treatment with PEM containing $4 \mathrm{~m}$ glycerol and $0.5 \%$ Triton X-100 for $10 \mathrm{~min}$ at $37^{\circ} \mathrm{C}$, and cells were double-stained with anti-tubulin (green in middle panels) and anti-Tau (red in left panels), followed by fluorescently labeled secondary antibodies. Merged images are shown in the right panels. Scale bar, $20 \mu \mathrm{m}$.

et al., 2008). In all three cases, there was wide codistribution of Tau and MT (Fig. 2), suggesting that Tau phosphorylation at the AT8 sites does not inhibit the binding of Tau to MTs in cells, as reported previously (Rankin et al., 2005; Hanger et al., 2009). Furthermore, in vitro studies indicated that both recombinant pure Tau 3A and 3D bound to MTs similarly to Tau WT (data not shown).

\section{Expression of Tau constructs in PC12 cells and cultured cortical neurons}

Expression levels of Tau WT, 3A, and 3D in PC12 cells and cultured neurons were measured after immunofluorescent staining. The immunostainings of neurons are shown in Figure 3A. The staining intensity increased with concentrations of plasmids from 2 to $3 \mu \mathrm{g}$ used for transfection (Fig. $3 B, C$ ). When $2.5 \mu \mathrm{g}$ of plasmids were used, the fluorescent intensities were $\sim 1.7$ and $\sim 1.5$ fold of untransfected PC12 cells (Fig. $3 B$ ) and cultured neurons (Fig. $3 C$ ), respectively. Expression levels were almost similar among Tau WT, 3A, and 3D constructs (Fig. $3 B, C$ ). The effect of Tau on mitochondrial movement was dependent on the amount of Tau plasmid used for transfection. While transfection with less than $2 \mu \mathrm{g}$ did not significantly affect mitochondrial movement, more than $3 \mu \mathrm{g}$ had a substantial (and similar) effect for all Tau constructs (data not shown). Differences were observed with 2.5 $\mu \mathrm{g}$ DNA, which was used in subsequent experiments.

Tau phosphorylation reduces mitochondrial motility in neuritic processes of PC12 cells

To determine the effect of Tau mutants on mitochondrial movement in parallel bundles of MTs, we first used NGF- treated PC12 cells, which have been previously employed for MT-mitochondria studies (Tatebayashi et al., 2004; Morel et al., 2010). We cotransfected Tau constructs and Mito-GFP into PC12 cells and observed the movement of GFPlabeled mitochondria in neurite-like processes $3 \mathrm{~d}$ after NGF treatment. Mitochondria were distributed throughout the processes of PC12 cells (left panels of Fig. 4A). Mitochondrial motility was recorded over $300 \mathrm{~s}$ in randomly selected processes, and examples are shown as kymographs in Figure $4 A$. The number of motile mitochondria was higher in control PC12 cells compared with Tau-overexpressing cells. The percentages of stationary phase of mitochondria, direction of movement, and velocity were quantified (Fig. $4 B-D$ ). The ratio of stationary phase was $23.0 \%$ in control PC12 cells but $47.6 \%$ in cells expressing Tau WT and $44.1 \%$ for $3 \mathrm{~A}$; for $3 \mathrm{D}$, however, the percentage was higher still, 61.3\% (Fig. 4B). Moving mitochondria in neurites overexpressing Tau 3D were significantly fewer than those in Tau WT or 3A-expressing neurites, indicating the stronger inhibitory activity of Tau 3D. Effect of Tau overexpression on anterograde and retrograde movements is shown in Figure $4 C$. Overexpression of each Tau construct specifically decreased the anterograde movement of mitochondria (compare control with WT/3A/3D in Fig. 4C). However, neither Tau 3D nor 3A had further preferential effect on the direction of mitochondrial movement (Fig. 4).

Tau overexpression also affected the velocity of mitochondrial movement in both directions. In control PC12 cells, the velocity was normally distributed with a peak at $0.2-0.3 \mu \mathrm{m} / \mathrm{s}$ (Fig. $4 D)$. The mean velocities were around $0.29 \pm 0.03$ and $0.31 \pm$ $0.04 \mu \mathrm{m} / \mathrm{s}$ for anterograde and retrograde movement, respectively. Tau overexpression shifted the peak to $<0.1 \mu \mathrm{m} / \mathrm{s}$. The percentages of the total anterograde- and retrograde-moving mitochondria moving $<0.1 \mu \mathrm{m} / \mathrm{s}$ were 53.3 and $57.7 \%$ in Tau WT-expressing PC12 cells, 54.2 and $62.6 \%$ in Tau $3 \mathrm{~A}-$ expressing PC12 cells, and 54.3 and $56.8 \%$ in Tau 3D-expressing PC12 cells, respectively. The mean velocities for anterograde and retrograde movement were reduced to $0.19 \pm 0.056$ and $0.17 \pm 0.035 \mu \mathrm{m} / \mathrm{s}$ by Tau WT overexpression, $0.20 \pm 0.02$ and $0.17 \pm 0.07 \mu \mathrm{m} / \mathrm{s}$ by Tau $3 \mathrm{~A}$ overexpression, and $0.22 \pm$ 0.06 and $0.18 \pm 0.04 \mu \mathrm{m} / \mathrm{s}$ by Tau 3D overexpression, respectively. Notably, for Tau 3D there was a second peak of mitochondrial movement with a velocity of $\sim 0.4-0.6 \mu \mathrm{m} / \mathrm{s}$, particularly in the anterograde direction. Thus, there may be a population of mitochondria that is less affected by Tau $3 \mathrm{D}$. The results that Tau overexpression increased the stationary phase of mitochondria are consistent with previous reports (Trinczek et al., 1999; Thies and Mandelkow, 2007; Stoothoff et al., 2009). Our novel finding was that phosphorylation mimic mutation (3D) at the AT8 Alzheimer sites had an additional effect on mitochondrial motility compared with Tau WT and 3A in neuron-like processes of PC12 cells. 


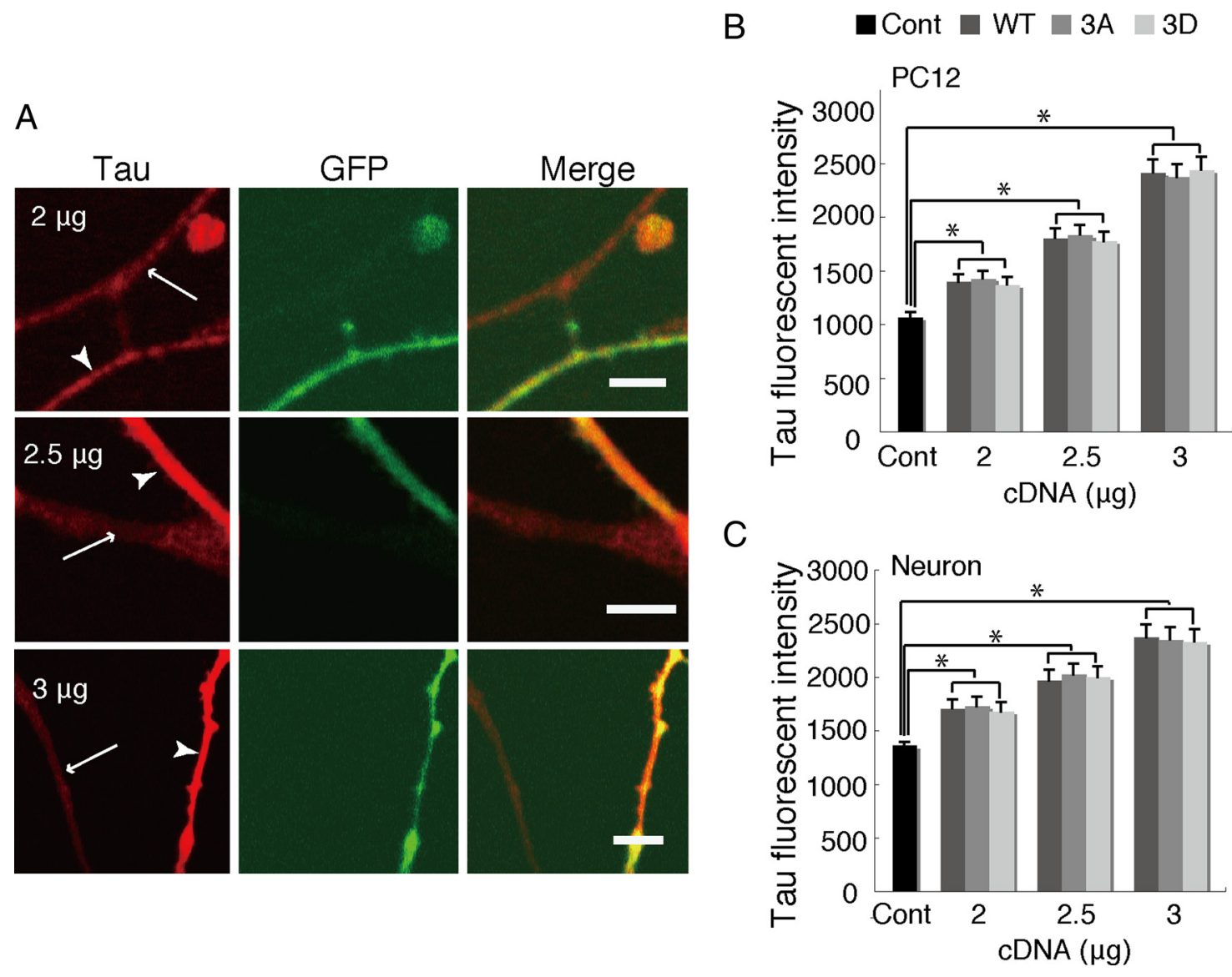

Figure 3. Quantification of levels of Tau overexpression in the PC12 cell neurites and in neuronal axons. A, Immunostaining of cortical neurons overexpressing Tau with anti-human Tau. Plasmids encoding Tau at concentrations of 2, 2.5, and $3 \mu \mathrm{m}$ were cotransfected with EGFP vector into cultured cortical neurons at DIV6, and these neurons were stained at DIV11. Transfected axons are indicated with arrowheads and un-transfected axons with arrows. Scale bar, $5 \mu \mathrm{m}$. $\boldsymbol{B}, \boldsymbol{C}$, Quantification of Tau expressed in PC12 cell neurites $(\boldsymbol{B})$ and axon of cortical neurons $(\boldsymbol{C})$. $(\boldsymbol{n}=20$ for each of PC12 cells and neurons, ${ }^{*} p<0.01$, one-way ANOVA). Cont, Control.

Increased pausing and decreased velocity of mitochondria in axons of cultured neurons expressing Tau 3D

Tau is predominantly expressed in neuronal axons. We therefore compared the effects of Tau 3D with those of Tau WT or 3A on mitochondrial movement in axons of cultured cortical neurons. Mitochondrial distribution in axons transfected with Tau and Mito-GFP are shown in left panels of Figure 5A. During the $300 \mathrm{~s}$ observation period, mitochondria exhibited complex motile behavior such as anterograde and retrograde movements with frequent pausing. The duration of stationary phase was $31.6 \%$ in control neurons, which was higher than that observed in PC12 cells, consistent with a previous report (Morel et al., 2010). A significant increase in stationary phase was observed after transfection with Tau WT $(48.5 \%)$ or $3 \mathrm{~A}(46.6 \%)$; however, a higher stationary state $(64.4 \%)$ was caused by Tau $3 \mathrm{D}$ (Fig. $5 B$ ). The stational phase induced by Tau 3D was significantly higher than those induced by Tau WT and 3A, indicating that Tau 3D has stronger inhibitory activity against mitochondrial movement. Direction-dependent inhibition of mitochondrial movement by Tau is summarized in Figure 5C. Tau protein dramatically reduced the population of anterograde-moving mitochondria, consistent with previous reports (Stamer et al., 2002; Dixit et al., 2008; Vershinin et al., 2008; Stoothoff et al., 2009). However, the ratio of mitochondria moving anterogradely and retrogradely was not different among three Tau constructs, indicating that phosphorylation at the AT8 sites does not affect the direction of mitochondrial movements.
Tau overexpression also reduced the velocity of mitochondria in axons. In control neurons, mitochondria showed a peak velocity of $0.2-0.3 \mu \mathrm{m} / \mathrm{s}$ in both directions, and a substantial proportion also moved faster than $0.6 \mu \mathrm{m} / \mathrm{s}$ (Fig. $5 D$ ). Tau overexpression shifted the peak to a slower rate, with peak velocities of $0.05-0.3 \mu \mathrm{m} / \mathrm{s}$, depending on the Tau construct. The relative ratio of mitochondria with velocities less than $0.3 \mu \mathrm{m} / \mathrm{s}$ in the anterograde and retrograde directions were 31.0 and $38.2 \%$ in control neurons, 88.3 and $84.4 \%$ in Tau WT-expressing neurons, 86.9 and $82.6 \%$ in Tau 3A-expressing neurons, and 84.7 and $84.4 \%$ in Tau $3 \mathrm{D}$-expressing neurons. The mean anterograde and retrograde velocities were $0.55 \pm 0.08$ and $0.45 \pm 0.03 \mu \mathrm{m} / \mathrm{s}$, respectively, in control cells, but they were reduced to $0.17 \pm 0.03$ and $0.18 \pm 0.06 \mu \mathrm{m} / \mathrm{s}$ in Tau WT-overexpressing neurons, $0.18 \pm 0.03$ and $0.19 \pm 0.07 \mu \mathrm{m} / \mathrm{s}$ in Tau 3A-overexpressing neurons, and $0.18 \pm 0.03$ and $0.18 \pm 0.04 \mu \mathrm{m} / \mathrm{s}$ in Tau 3Doverexpressing neurons. Velocities faster than $1 \mu \mathrm{m} / \mathrm{s}$, which accounted for 15 and $11 \%$ of anterograde and retrograde movements, respectively, in control neurons, were not observed in any Tau-overexpressing neurons. As in PC12 cells, neurons expressing Tau 3D had a small proportion of mitochondria that moved at $\sim 0.5-0.6 \mu \mathrm{m} / \mathrm{s}$, particularly in the anterograde direction, whereas neurons expressing Tau WT or 3A did not. Thus, Tau with phosphorylation mimic mutation (3D) at the AT8 sites suppressed mitochondrial movement in axons of cultured cortical neurons more than Tau WT or 3A. 
A
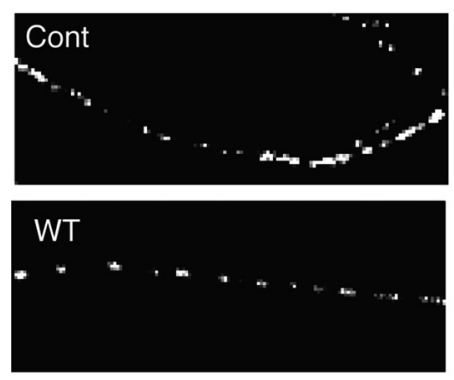

B
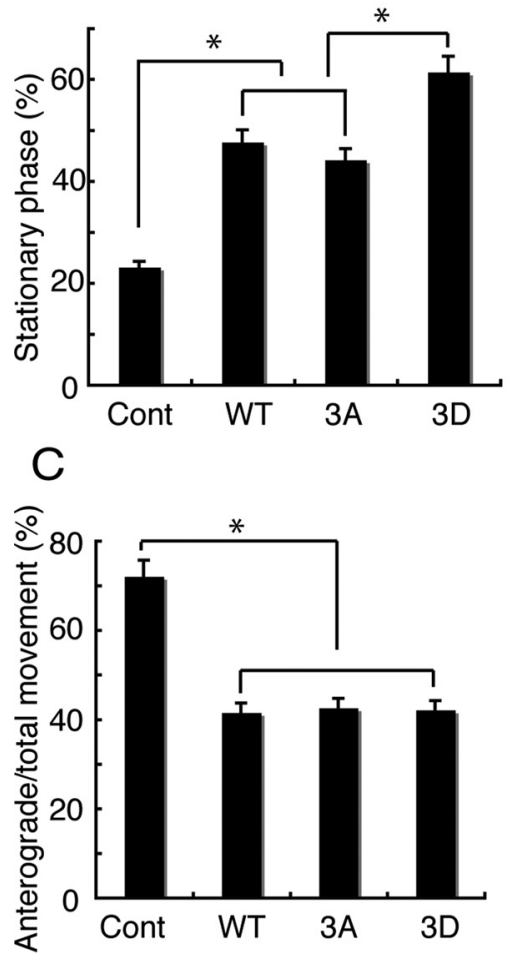
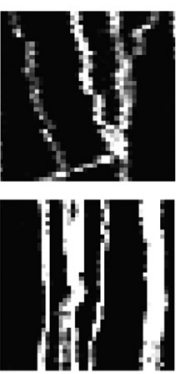

D
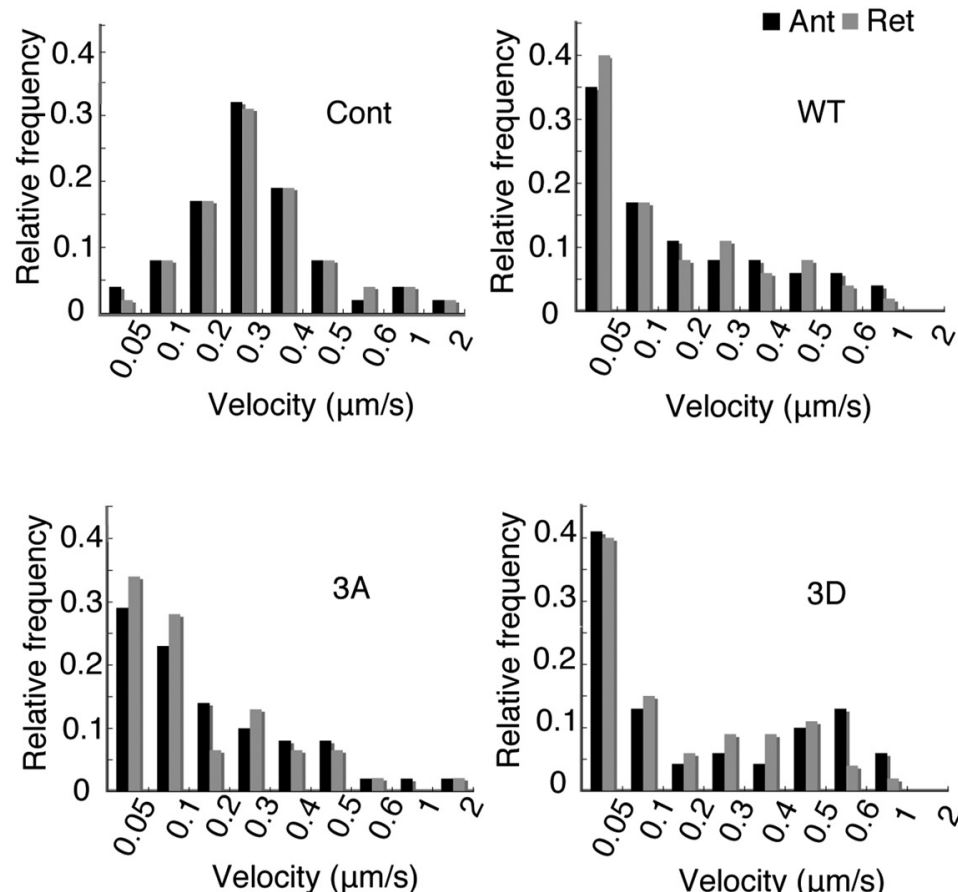

Antero $\longrightarrow$
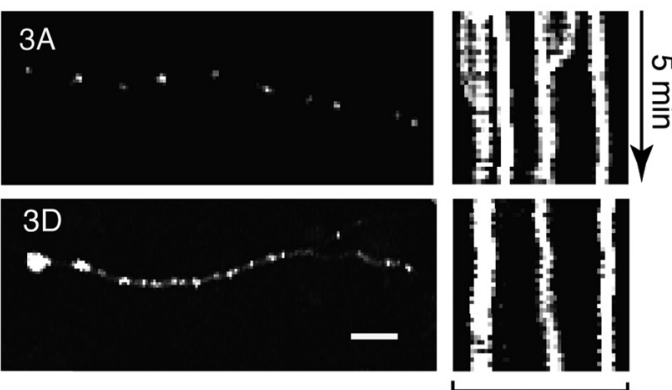

$50 \mu \mathrm{m}$

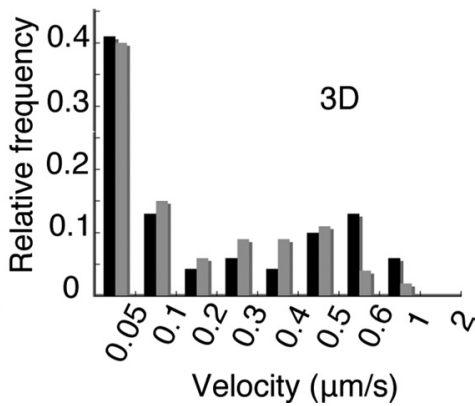

Figure 4. Effects of Tau WT, $3 D$, or $3 \mathrm{~A}$ on mitochondrial movement in $\mathrm{PC} 12$ cells. $A$, Mitochondrial distribution and movements in neurite process of $\mathrm{PC} 12$ cells. $\mathrm{PC} 12$ cells were transfected with a Mito-GFP vector alone as a control (Cont) or cotransfected with a Mito-GFP and Tau WT, 3A, or $3 \mathrm{D}$ vector. PC12 cells were treated with NGF for $72 \mathrm{~h}$ after transfection. Mitochondrial distribution is shown by fluorescence of Mito-GFP (left panels). Right panels are kymographs of mitochondria moving in a process of a PC12 cell. Antero, Anterograde. Bar, $20 \mu \mathrm{m}$. $\boldsymbol{B}$, The percentage ratio of pausing mitochondria in $\mathrm{PC} 12$ cell processes. Mitochondrial movement was recorded in the neurite-like processes at $5 \mathrm{~s}$ intervals over a $300 \mathrm{~s}$ period. Any mitochondrion that translocated at least $0.1 \mu \mathrm{m}$ between two image frames was considered to be moving. The pausing time was expressed as the percentage of the total observation time $\left(n=35,{ }^{*} p<0.01\right.$, one-way ANOVA). C, Effect of Tau WT, $3 \mathrm{~A}$, or $3 \mathrm{D}$ on anterograde or retrograde movement of mitochondria. The vertical axis indicates the ratio of anterogradely moving time to total moving duration. Statistical analysis was performed by ANCOVA as described in Materials and Methods. The ratio was significantly different between control and Tau-overexpressing $\mathrm{PC} 12$ cells $\left({ }^{*} p<0.01\right)$, but was not significant between three Tau constructs $(p=0.75)$. D, Effect of Tau WT, 3D, or 3A on the velocity of mitochondria. Relative frequency of mitochondria moving at the indicated velocities in the anterograde (Ant, black) or retrograde (Ret, gray) direction for control, WT, 3A, or 3D. $n=35$ mitochondria per sample.

The binding of kinesin to MTs is not affected by Tau in any phosphorylation state

Tau impairs mitochondrial movement by inhibiting the interaction of kinesin with MTs (Hagiwara et al., 1994). To delineate whether the reduced mitochondrial movement caused by Tau 3D expression was due to increased inhibition of the motor-MT interaction by Tau $3 \mathrm{D}$, we examined the binding of the kinesin motor domain fragment RK430 to MTs in the presence of Tau WT, 3A, or 3D. We used the head domain of kinesin because the tail domain also has a MTbinding domain, which competes with Tau in in vitro MT-binding experiments (Seeger and Rice, 2010). Histidine-tagged kinesin (kinesin-His) that was bound or not bound to MTs was detected by Coomassie staining (Fig. 6A) and Western blotting (Fig. 6B) of the MT pellets and supernatants after centrifugation. Increasing the amount of Tau WT or Tau mutants did not alter the binding of RK430 kinesin-His to MTs even though excess Tau was added such that unbound Tau appeared in the supernatant. Thus, the reduced mitochondrial movement was not caused by inhibiting the interaction between the kinesin head domain and MTs.

The phosphorylation mimic Tau 3D increases MT spacing in processes of Sf9 cells

Tau is a space-making protein between MTs (Chen et al., 1992; Frappier et al., 1994; Marx et al., 2000). We examined the effect of phosphorylation at the AT8 sites on MT spacing in vitro. MTs were polymerized in the presence of Tau and pelleted by centrifugation. Thin-section electron micrographs of MTs cut perpendicularly to the MT axis are shown in Figure 7A-C. The inter-MT distances were 
A
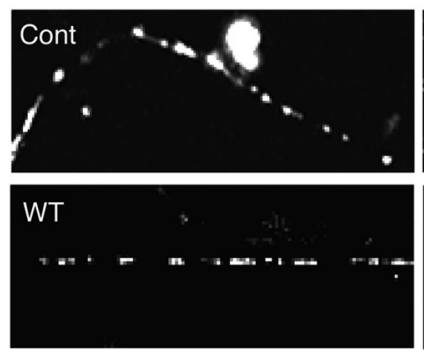

B
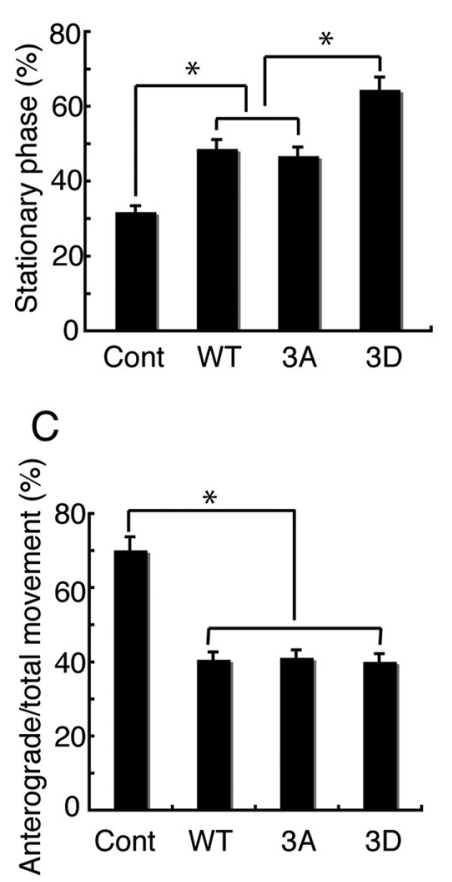

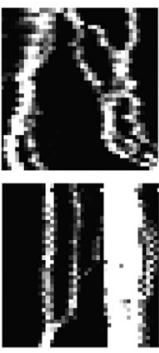

D
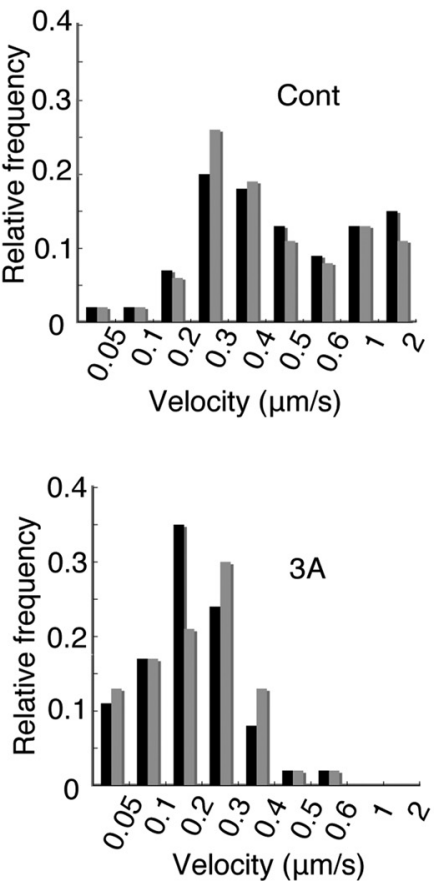

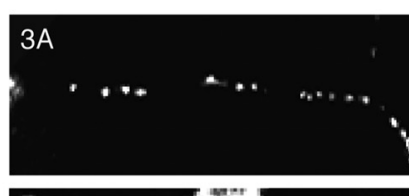

Antero $\longrightarrow$
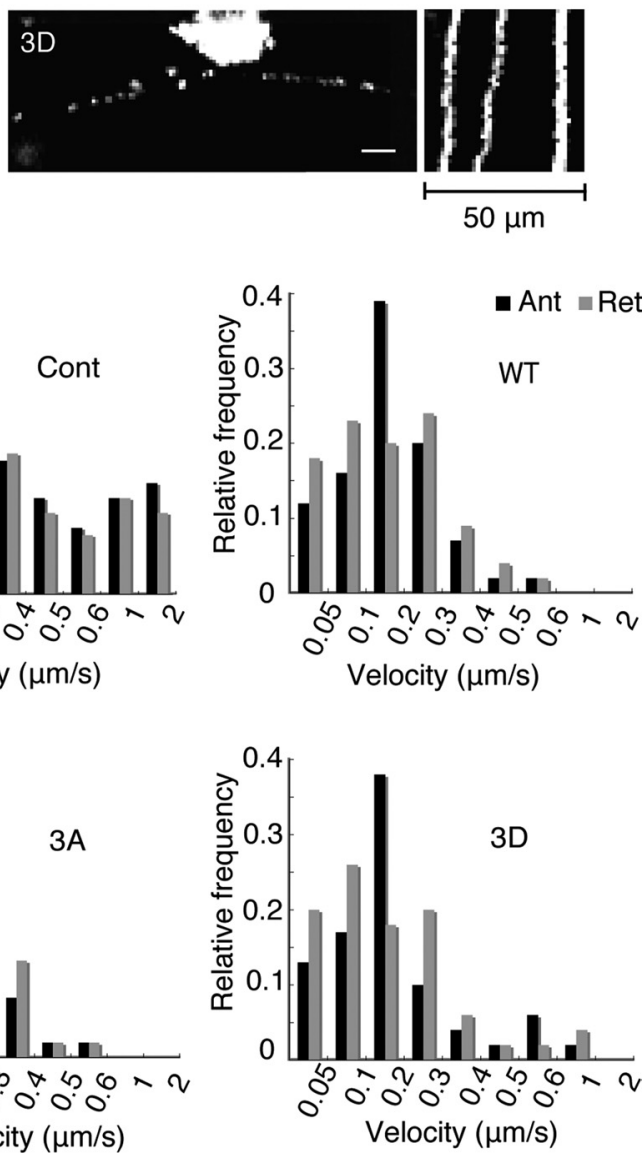

Figure 5. Reduced motile behavior of mitochondria in Tau-overexpressing neurons. $A$, Mitochondrial distribution and movements in axons of neurons. Cultured neurons at DIV6 were transfected with Mito-GFP vector alone (Cont, Control) or cotransfected with Mito-GFP and Tau WT, Tau 3A, or Tau 3D vector. Mitochondrial distribution is shown by fluorescence of Mito-GFP (left panels). Bar, 20 um. Right panels are kymographs of mitochondria moving in axon of neurons. Antero, Anterograde. $\boldsymbol{B}$, The percentage ratio of pausing mitochondria in axon of cultured neurons. The pausing duration was expressed as the percentage of total observation period $\left(n=35,{ }^{*} p<0.01\right.$, one-way ANOVA,).C, The effect of Tau mutants on anterograde or retrograde movement of mitochondria. The vertical axis is the ratio of anterogradely moving duration to total moving period. The results were analyzed statistically by ANCOVA as described in Materials and Methods. The ratio was significantly different between control and Tau-overexpressing neurons ( $p<0.01$ ) but was not different between three Tau constructs ( $p=0.55)$. D, Effect of Tau mutants on the velocity of mitochondria. Relative frequencies of mitochondria moving at the indicated velocities are shown in control neurons or in neurons expressing Tau WT, Tau $3 \mathrm{~A}$, or Tau 3D. Black represents anterograde (Ant) movement, and gray represents retrograde (Ret) movement ( $n=35$ mitochondria per sample).

A
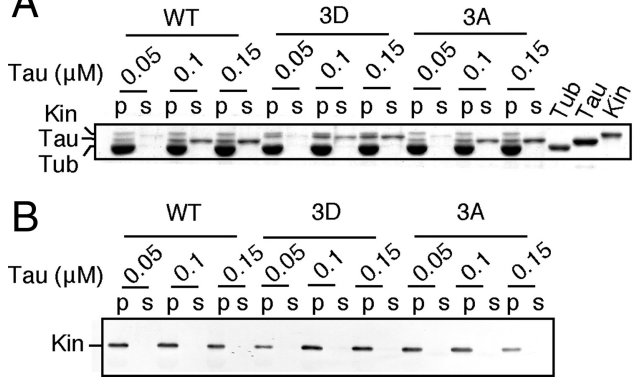

Figure 6. Tau does not inhibit the binding of kinesin to MTs. A, Coomassie staining of an SDS-PAGE gel to show the binding of kinesin to MTs independent of Tau-binding. MTs polymerized in the presence of $0.05,0.1$, or $0.15 \mu \mathrm{m}$ Tau WT, 3A, or 3D were incubated with $0.1 \mu \mathrm{m}$ kinesin head domain-His and, after separation of MTs by centrifugation, the MT pellet (p) and supernatant (s) were subjected to SDS-PAGE. The right side three lanes are tubulin (Tub), Tau WT (Tau), and kinesin (Kin) head domain-His, respectively, for references. $\boldsymbol{B}$, An immunoblot confirming the binding of kinesin to MTs. MT pellet (p) and supernatant (s) shown in $\boldsymbol{A}$ are immunoblotted with anti-His antibody for detection of kinesin head domain-His. measured from wall to wall of nearest-neighbor MTs. The distances were typically $<10 \mathrm{~nm}$ in the MT pellets polymerized with Tau WT, $3 \mathrm{~A}$, and $3 \mathrm{D}$ (Fig. $7 \mathrm{D}$ ), indicating that the phosphorylationmimicking mutation at Ser199/Ser202/Thr205 did not affect the inter-MT spacing in pelleted MTs.

To determine whether this was the case in cells, we employed an Sf 9 cell overexpression system that was used for Tau-induced MT bundle formation (Kanai et al., 1989; Frappier et al., 1994). We measured the distances between the nearest-neighbor MTs in the MT bundles formed in the process of Sf9 cells after infection of cells with baculovirus encoding Tau. Electron micrographs of processes in which most MTs were cut perpendicularly are shown in Figure 7, $E-G$, and the inter-MT distances are shown in Figure $7 H$. Of the 85 inter-MT distances measured, there was no significant difference between cells infected with the various Tau proteins (Fig. $7 \mathrm{H}$ ). Because only a small number of processes were formed by Tau overexpression alone, however, the number of inter-MT distances we counted was not sufficient. 

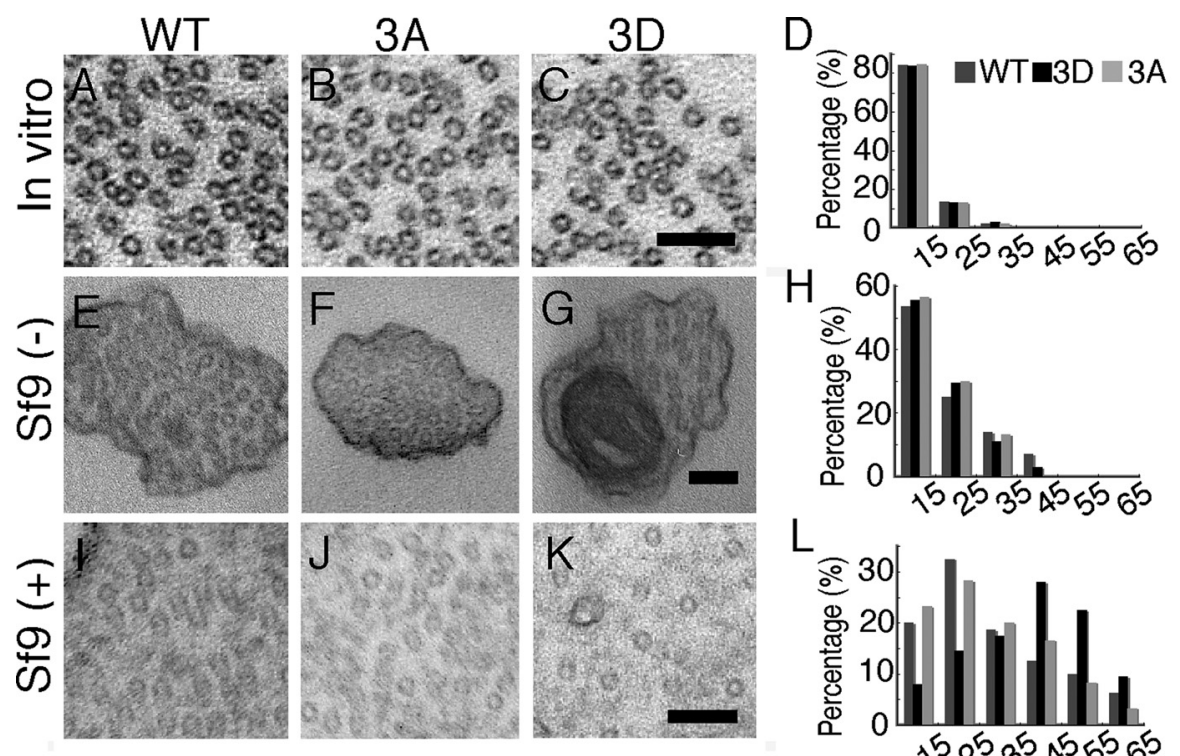

M

$\mathrm{N}$
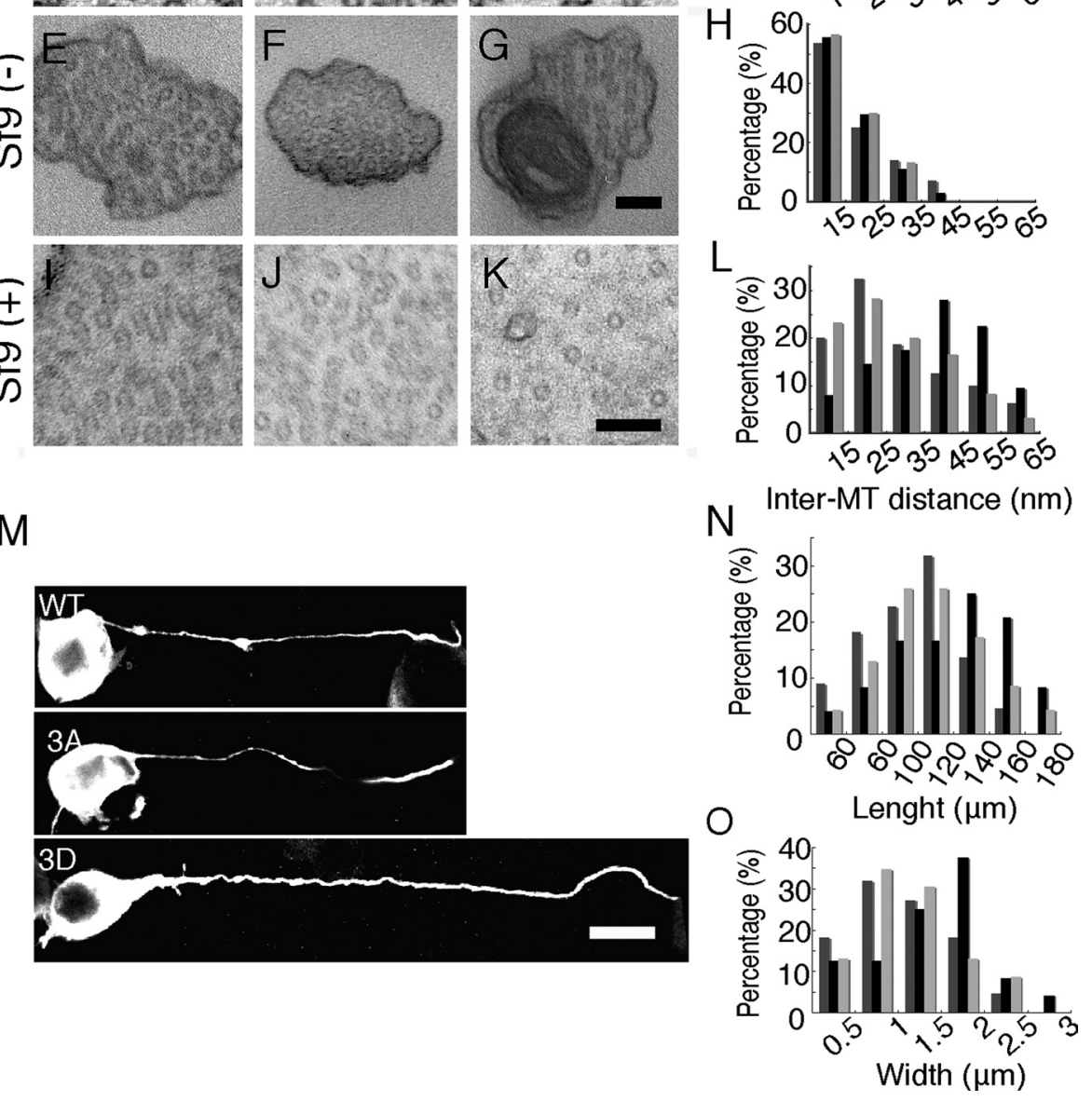

Figure 7. Inter-MT distance in MT bundles in the presence of Tau mutants. $\boldsymbol{A}-\boldsymbol{C}$, Electron micrographs of cross-sections of MT pellets polymerized in vitro with Tau WT (A), 3A (B), or 3D (C). Scale bar, $100 \mathrm{~nm}$. D. The wall-to-wall distances between nearest-neighbor MTs were measured and expressed as the percentage of the total number of counted MTs. The mean distance was $11.7 \pm 1.7$ $\mathrm{nm}$ for Tau WT $(n=48), 11.8 \pm 2.1 \mathrm{~nm}$ for Tau 3A $(n=51)$, and $11.9 \pm 1.6 \mathrm{~nm}$ for Tau 3D $(n=69)$. $\boldsymbol{E}-\boldsymbol{G}$, Electron micrographs of processes of Sf9 cells [untreated $(-)$ ] expressing Tau WT (E), $3 \mathrm{~A}(\boldsymbol{F})$, or $3 \mathrm{D}(\boldsymbol{G})$. $\boldsymbol{H}$, Inter-MT distances were measured between nearest-neighbor MTs and expressed as the percentage of the total number of counts. The mean distance was $15.7 \pm 2.2 \mathrm{~nm}$ for Tau WT $(n=30), 17.5 \pm 2.7 \mathrm{~nm}$ for Tau 3A ( $n=27)$, and $16.3 \pm 2.2 \mathrm{~nm}$ for Tau 3D $(n=28) . I-K$, Electron micrographs of processes of latrunculin B-treated $(+)$ Sf9 cells expressing Tau WT $(I)$, $3 \mathrm{~A}(\boldsymbol{J})$, or $3 \mathrm{D}(\boldsymbol{K})$. Sf9 cells were treated with $0.5 \mu \mathrm{g} / \mathrm{ml}$ latrunculin B for $72 \mathrm{~h}$ after infection with baculovirus expressing each Tau. $L$, Inter-MT distances were measured and expressed as the relative ratio of the total number of counts. The mean distance was $26.8 \pm 2.7 \mathrm{~nm}$ for Tau WT $(n=60), 27.2 \pm 2.0 \mathrm{~nm}$ for Tau $3 \mathrm{~A}(n=80)$, and $37.1 \pm 2.3 \mathrm{~nm}$ for Tau 3D ( $n=75)$. $\boldsymbol{M}$, Immunostaining of Sf9 cells overexpressing Tau 3D (top), Tau 3A (middle), or WT (bottom) with anti-Tau. Scale bar, $20 \mu \mathrm{m} . \mathbf{N}, \mathbf{O}$, Sf9 cells overexpressing Tau 3D formed longer and wider processes compared to those expressing Tau WT or 3A. $\boldsymbol{N}$, The length distribution of Sf9 cell processes overexpressing Tau constructs. The mean length was $106.5 \pm 3.7 \mu \mathrm{m}$ for Tau WT, $97.2 \pm 5.3 \mu \mathrm{m}$ for Tau $3 \mathrm{~A}$, and $119.1 \pm$ $4.3 \mu \mathrm{m}$ for Tau 3D ( $n=20$ for each Tau construct). 0 , The width distribution of Sf9 cell processes overexpressing Tau constructs. The mean width was $1.09 \pm 0.04 \mu \mathrm{m}$ for Tau WT, $1.04 \pm 0.09 \mu \mathrm{m}$ for Tau 3A, and $1.39 \pm 0.07 \mu \mathrm{m}$ for Tau 3D ( $n=20$ for each Tau construct).

We therefore treated Tau-overexpressing Sf 9 cells with latrunculin B, which disrupts actin filaments, to increase the number of processes (Knowles et al., 1994). When the processes were stained with anti-Tau antibody, we noticed that Sf9 cells expressing Tau $3 \mathrm{D}$ had larger and longer processes than cells expressing Tau WT or $3 \mathrm{~A}$ (Fig. $7 \mathrm{M}-\mathrm{O})$. The mean length was $106.5 \pm 3.7 \mu \mathrm{m}$ for Tau WT, $97.2 \pm 5.3 \mu \mathrm{m}$ for Tau 3A, and $119.1 \pm 4.3 \mu \mathrm{m}$ for Tau 3D (Fig. $7 N$ ). The mean diameter was $1.09 \pm 0.04 \mu \mathrm{m}$ for Tau WT, $1.04 \pm 0.09 \mu \mathrm{m}$ for Tau 3A, and 1.39 $\pm 0.07 \mu \mathrm{m}$ for Tau 3D (Fig. $7 O)$. We then observed MTs in processes by electron microscopy (Fig. $7 I-K)$. The inter-MT distances were greater in Tau 3Doverexpressing cells compared to those overexpressing WT or 3A (Fig. $7 \mathrm{~L}$ ). Although most distances fell in the range of $15-25 \mathrm{~nm}$ in Tau WT- and 3A-expressing cells (mean distance $=26.8 \pm 2.7$ and $27.2 \pm 2.0 \mathrm{~nm}$, respectively), almost same as the previous results (Frappier et al., 1994), that range was $35-45 \mathrm{~nm}$ in Tau $3 \mathrm{D}$-expressing cells $($ mean distance $=37.1 \pm 2.3 \mathrm{~nm})($ Fig. $7 L)$. Because latrunculin B reduces membrane tension by depolymerizing submembranous actin filaments, these results suggested that, under reduced tension, phosphorylation of Tau at the AT8 sites increases the space between MTs.

Tau overexpression reduces the inter-MT distances in neurites of PC12 cells

We wanted to know how expression of Tau 3A or 3D affects the inter-MT distance in axons or neuritic processes. We performed 
the experiments with PC12 cells in which mitochondrial movements were affected by Tau expression as was observed in neuronal axons. Typical electron micrographs are shown in Figure 8A. More MTs were found in neurites of $\mathrm{PC} 12$ cells overexpressing Tau. The inter-MT distances were reduced to $\sim 35 \mathrm{~nm}$ in Tauexpressing processes from $\sim 45 \mathrm{~nm}$ in the control untransfected processes (Fig. $8 \mathrm{~B}$ ). In contrast, there was no significant difference in the inter-MT distances among Tau WT, 3A, and 3D overexpression (Fig. $8 B)$. The mean distance was $33.1 \pm 2.4$ $\mathrm{nm}$ for Tau WT, $34.1 \pm 2.6 \mathrm{~nm}$ for Tau $3 \mathrm{~A}$, and $34.19 \pm 2.8$ for $3 \mathrm{D}$.

\section{Discussion}

Tau is a major MAP in axons and plays a role in regulating organelle transport and the dynamics of axonal MTs. Many reports have described the inhibition of mitochondrial transport by overexpressing Tau (Ebneth et al., 1998; Trinczek et al., 1999; Stamer et al., 2002; Dixit et al., 2008; Dubey et al., 2008; Stoothoff et al., 2009; Vossel et al., 2010). However, the molecular mechanism has not been determined. We studied the effect of AT8 Alzheimer phosphorylation (Ser199/Ser202/ Thr205) of Tau on mitochondrial movement and found that the phosphorylation mimetic form, Tau 3D, inhibited mitochondrial transport to a greater degree than Tau WT and Tau 3A. Based on these findings together with the observation that the inter-MT distance was greater in MT bundles containing Tau 3D, we would like to propose that phosphorylation of Tau at the AT8 sites affects the transport of mitochondria along MTs by changing the inter-MT spaces.

Tau is a phosphoprotein with multiple phosphorylation sites; mass spectroscopic analysis indicates ten and five major sites in fetal and adult rat brains, respectively (Watanabe et al., 1993; MorishimaKawashima et al., 1995; Planel et al., 2002). Major phosphorylation sites are in the Ser-Pro and Thr-Pro sequences, and most of them are in the region flanking the MT-binding domain. Phosphorylation at these sites reduces, but does not abolish, Tau binding to MTs, leading to more dynamic MTs (Wada et al., 1998; Liu et al., 2007). However, site-specific functions have not been completely investigated. Among the many Ser/Thr phosphorylation sites, Ser199, Ser202, and Thr205, which contain the recognition epitope for the AT8 monoclonal antibody, are particularly interesting. The AT8 sites are not only physiological phosphorylation sites (Kimura et al., 2007; Verwer et al., 2007) but also markers of hyperphosphorylation in Alzheimer's disease (Plattner et al., 2006). The Ser199/Ser202/Thr205 sites are present in the border between the $\mathrm{N}$-terminal projection region and the MT-binding region. According to the paperclip structural model of Tau (Jeganathan et al., 2006), the site near Ser199/Ser202/Thr205 folds and the $\mathrm{N}$-terminal region is situated close to the MT-binding repeats. When the Ser199/Ser202/Thr205 sites are phosphorylated the $\mathrm{N}$-terminal domain swings away from the C-terminal domain, resulting in a conformation that extends from the MT wall (Jeganathan et al., 2008). The extended projection may increase the distance between MTs, although this idea has not been validated. By observing MT bundles in Sf9 cell processes treated with latrunculin $\mathrm{B}$, we found that the inter-MT distance in MT
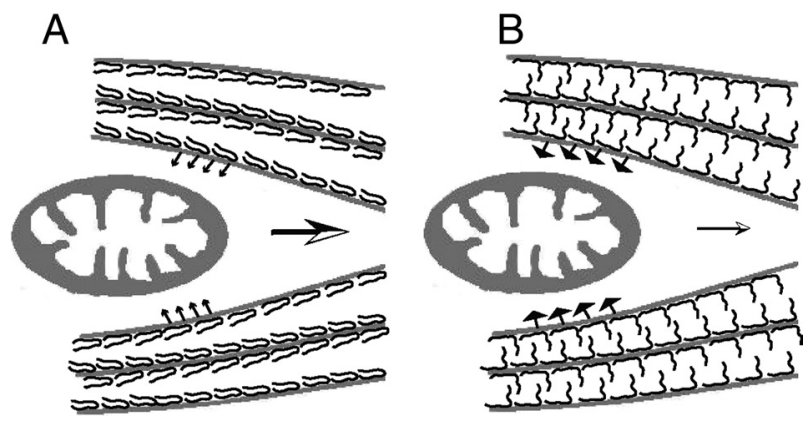

Figure 9. Schematic representation of the greater impact of Tau phosphorylation on mitochondrial transport compared to nonphosphorylated Tau. $\boldsymbol{A}$, Tau is a space-making protein attached to the MT surface. When Tau is not phosphorylated at Ser199/Ser202/Thr205, the $\mathrm{N}$-terminal domain is folded. This conformation does not produce much resistance force against mitochondrial movement because MTs are in relatively close proximity. $\boldsymbol{B}$, Tau phosphorylation at Ser199/Ser202/Thr205 may extend the projection domain away from the MT surface, increasing the repulsive forces between MTs. In this conformation, mitochondria encounter greater MT resistance to the slowing or arresting of their movement.

bundles containing Tau 3D was longer than that in MT bundles containing Tau WT or Tau 3A.

Tau overexpression inhibits mitochondrial movement in various cell types (Ebneth et al., 1998; Trinczek et al., 1999; Stamer et al., 2002; Dixit et al., 2008; Stoothoff et al., 2009; Vossel et al., 2010). We also observed an inhibition of mitochondrial movement in PC12 cells and cortical neurons. Tau WT overexpression increased the pausing frequency from 31.6 to $48.5 \%$ in neurons, which is almost identical to previous results (Stamer et al., 2002). Overexpression of either Tau WT, 3A, or 3D reduced anterograde movement of mitochondria in PC12 cells and cortical neurons, as reported (Mandelkow et al., 2004; Hollenbeck and Saxton, 2005; Dixit et al., 2008). Furthermore, the velocity was reduced similarly in both directions by Tau overexpression regardless of its phosphorylation state, although the inhibition profile differed slightly among the three Tau constructs. Thus, Tau clearly inhibits mitochondrial transport independent of its phosphorylation state. Several models have been proposed for Tau-mediated inhibition of mitochondrial transport: overstabilization of MTs (Shemesh et al., 2008), competition between motor proteins for interaction with the MT surface (Hagiwara et al., 1994), and in- 
hibition of motor protein access to MTs (Seeger and Rice, 2010). Another mechanism proposed recently involves the distance between MTs (Thies and Mandelkow, 2007). When Tau is overexpressed in cortical neurons, tubulin synthesis is upregulated and MTs become more numerous and densely packed, resulting in inhibition of mitochondrial movement. This observation was in dendrites, but similar Tau overexpression-induced increases in MTs were reported in axons (Sudo and Baas, 2010). We observed here that Tau overexpression increased the number of MTs and reduced the inter-MT spaces in neurites of PC12 cells. Our observation is consistent with the last model described above. Considering that mitochondria would be transported within MT bundles in cultured neurons ( $\mathrm{Yu}$ and Baas, 1994; Rochlin et al., 1996), limited spacing between MTs may block mitochondrial movement in neuritic processes such as axon and dendrites.

We found that Tau 3D more potently inhibited mitochondrial transport than Tau WT or Tau 3A. Phosphorylation of Tau at the AT8 sites has an additional inhibitory action on mitochondrial movement over the Tau molecule itself. We hypothesize that the inhibition caused by Ser199/Ser202/Thr205 phosphorylation relates to the distance between MTs. The inter-MT distance was the same (15-25 nm) in MT bundles formed by Tau WT, 3A, and 3D in Sf9 cell processes, but it was expanded to $35-45 \mathrm{~nm}$ in processes expressing Tau 3D when actin filaments were disrupted by latrunculin B. Actin filaments are abundant in submembranous regions, providing tension to plasma membranes. As reported (Knowles et al., 1994), disassembly of actin filaments increases the number of processes induced by Tau overexpression. Phosphorylation-dependent expansion of the space between MTs was observed only with reduced membrane tension. The force produced by outward extension of the projection domain may not be strong enough to push surrounding MTs against the membrane, which may explain why phosphorylation-induced expansion of the inter-MT distance has not been reported.

Axons are long processes that extend $\sim 1 \mathrm{~m}$ or more. To maintain axonal structures to over 80 years in humans, the axoplasm is filled with cytoskeletal components, and the outer surface is surrounded by thick myelin. These features may indicate that the axoplasmic MT milieu is under the strong tension (Yu and Baas, 1994; Rochlin et al., 1996). Phosphorylation of Tau at the AT8 sites tends to increase the inter-MT distance, but under strong tension the inter-MT distance cannot expand (Fig. 8), and instead the repulsive forces between MTs increase (Fig. 9). Increased repulsive forces between MTs would generate a stronger reactive resistance against mitochondria moving inside MT bundles. The AT8 sites are physiological sites for phosphorylation, but they are not always phosphorylated (Kimura et al., 2007; Verwer et al., 2007). The AT8 sites may be interconverted between phosphorylated and dephosphorylated states depending on the cell's need for mitochondrial movement. Dephosphorylation ahead of moving mitochondria would reduce the repulsive force between adjacent MTs for mitochondrial passage, and rephosphorylation of Tau behind mitochondria may facilitate the directional movement of mitochondria (Shahpasand et al., 2008). Of course, phosphorylation-dependent tunnel opening and closing for mitochondrial movement is expected to be coordinated with activities of motor proteins, protein kinases, and protein phosphatases. This is our working hypothesis, which we will explore further in the future.

We used PC12 cells and cultured cortical neurons in this study in which MTs are major cytoskeletal components. In matured or aged neurons, however, Tau may not be the only space-making protein that affects mitochondrial movement in a phosphory- lation-dependent manner. The C-terminal tail domains of neurofilament $\mathrm{M}$ and $\mathrm{H}$ subunits extrude outward from core filaments, as does the projection domain of Tau, to make spaces between neurofilaments (NFs) (Hisanaga and Hirokawa, 1989). NFs are highly phosphorylated in aged axons and AD neurodegenerative disease (Uchida et al., 2004; Rudrabhatla et al., 2010). Hyperphosphorylation of the tail domains would increase the inter-NF distances to expand the NF domain in axons (Kumar et al., 2002; Kanungo et al., 2011), giving higher pressure to the MT domain. This would suppress mitochondrial transport along MTs by restricting radial displacement of MTs. Thus, mitochondrial movements in aged and neurodegenerative axons would be affected in a more complicated manner.

AT8 reactivity has been frequently used as an indicator of hyperphosphorylation of Tau in AD brains or other tauopathies (Stoothoff and Johnson, 2005; Hanger et al., 2009). Impairment of mitochondrial traffic is also a feature of tauopathies (Stokin et al., 2005; Lippens et al., 2007). An unanswered issue is whether abnormal Tau phosphorylation is caused by impaired organelle trafficking or if blocked transport is a consequence of abnormal phosphorylation. Using the phosphorylation mimic Tau $3 \mathrm{D}$, we showed that Tau phosphorylation within the AT8 sites inhibited mitochondrial transport more effectively than in experiments carried out with Tau WT and Tau 3A. Because Tau $3 \mathrm{~A}$ and $3 \mathrm{D}$ have similar phosphorylation profiles at other sites, the observed effect is likely caused by phosphorylation at Ser199/Ser202/Thr205. Thus, our results suggest that the increased phosphorylation of the AT8 sites in brains of Alzheimer's patients decreases mitochondrial transport in axons, leading to axonal degeneration. Our current study not only leads us to focus on the AT8 sites with regard to Alzheimer's therapeutics but also indicates the effectiveness of a similar strategy addressing other abnormal phosphorylation sites on mitochondrial movement.

\section{References}

Bereiter-Hahn J, Jendrach M (2010) Mitochondrial dynamics. Int Rev Cell Mol Biol 284:1-65.

Bershadsky AD, Gelfand VI, Svitkina TM, Tint IS (1978) Microtubules in mouse embryo fibroblasts extracted with Triton X-100. Cell Biol Int Rep 2:425-432.

Chen J, Kanai Y, Cowan NJ, Hirokawa N (1992) Projection domains of MAP2 and tau determine spacings between microtubules in dendrites and axons. Nature 360:674-677.

Darios F, Muriel MP, Khondiker ME, Brice A, Ruberg M (2005) Neurotoxic calcium transfer from endoplasmic reticulum to mitochondria is regulated by cyclin-dependent kinase 5-dependent phosphorylation of tau. J Neurosci 25:4159-4168.

Dixit R, Ross JL, Goldman YE, Holzbaur EL (2008) Differential regulation of dynein and kinesin motor proteins by tau. Science 319:1086-1089.

Dubey M, Chaudhury P, Kabiru H, Shea TB (2008) Tau inhibits anterograde axonal transport and perturbs stability in growing axonal neurites in part by displacing kinesin cargo: neurofilaments attenuate taumediated neurite instability. Cell Motil Cytoskeleton 65:89-99.

Ebneth A, Godemann R, Stamer K, Illenberger S, Trinczek B, Mandelkow E (1998) Overexpression of tau protein inhibits kinesin-dependent trafficking of vesicles, mitochondria and endoplasmic reticulum: implications for Alzheimer's disease. J Cell Biol 143:777-794.

Endo R, Saito T, Asada A, Kawahara H, Ohshima T, Hisanaga S (2009) Commitment of 1-methyl-4-phenylpyrinidinium ion-induced neuronal cell death by proteasome-mediated degradation of p35 cyclin-dependent kinase 5 activator. J Biol Chem 284:26029-26039.

Frappier TF, Georgieff IS, Brown K, Shelanski ML (1994) Tau Regulation of microtubule-microtubule spacing and bundling. J Neurochem 63:2288-2294.

Furuta K, Edamatsu M, Maeda Y, Toyoshima YY (2008) Diffusion and di- 
rected movement: in vitro motile properties of fission yeast kinesin-14 Pkl1. J Biol Chem 283:36465-36473.

Goedert M, Jakes R, Vanmechelen E (1995) Monoclonal antibody AT8 recognises tau protein phosphorylated at both serine 202 and threonine 205. Neurosci Lett 189:167-169.

Hagiwara H, Yorifuji H, Sato-Yoshitake R, Hirokawa N (1994) Competition between motor molecules (kinesin and cytoplasmic dynein) and fibrous microtubule-associated proteins in binding to microtubules. J Biol Chem 269:3581-3589.

Hanger DP, Anderton BH, Noble W (2009) Tau phosphorylation: the therapeutic challenge for neurodegenerative disease. Trends Mol Med 15:112-119.

Hisanaga S, Hirokawa N (1989) The effects of dephosphorylation on the structure of the projections of neurofilament. J Neurosci 9:959-966.

Hollenbeck PJ, Saxton WM (2005) The axonal transport of mitochondria. J Cell Sci 118:5411-5419.

Hosokawa T, Saito T, Asada A, Fukunaga K, Hisanaga S (2010) Quantitative measurement of in vivo phosphorylation states of Cdk 5 activator $\mathrm{p} 35$ by Phos-tag SDS-PAGE. Mol Cell Proteomics 9:1133-1143.

Ishiguro K, Takamatsu M, Tomizawa K, Omori A, Takahashi M, Arioka M, Uchida T, Imahori K (1992) Tau protein kinase I converts normal tau protein into A68-like component of paired helical filaments. J Biol Chem 267:10897-10901.

Jeganathan S, von Bergen M, Brutlach H, Steinhoff HJ, Mandelkow E (2006) Global hairpin folding of tau in solution. Biochemistry 45:2283-2293.

Jeganathan S, Hascher A, Chinnathambi S, Biernat J, Mandelkow EM, Mandelkow E (2008) Proline-directed pseudo-phosphorylation at AT8 and PHF1 epitopes induces a compaction of the paperclip folding of tau and generates a pathological (MC-1) conformation. J Biol Chem 283:32066-32076.

Jicha GA, Bowser R, Kazam IG, Davies P (1997) Alz-50 and MC-1, a new monoclonal antibody raised to paired helical filaments, recognize conformational epitopes on recombinant tau. J Neurosci Res 48:128-132.

Jicha GA, Berenfeld B, Davies P (1999) Sequence requirements for formation of conformational variants of tau similar to those found in Alzheimer's disease. J Neurosci Res 55:713-723.

Kaminosono S, Saito T, Oyama F, Ohshima T, Asada A, Nagai Y, Nukina N, Hisanaga S (2008) Suppression of mutant Huntingtin aggregate formation by Cdk5/p35 through the effect on microtubule stability. J Neurosci 28:8747-8755.

Kanai Y, Takemura R, Oshima T, Mori H, Ihara Y, Yanagisawa M, Masaki T, Hirokawa N (1989) Expression of multiple tau isoforms and microtubule bundle formation in fibroblasts transfected with a single tau cDNA. J Cell Biol 109:1173-1184.

Kanungo J, Zheng Y, Rudrabhatla P, Amin N, Mishra B, Pant H (2011) Deregulation of cytoskeletal protein phosphorylation and neurodegeneration. In: Advances in neurobiology: cytoskeleton of the nervous system (Nixon RA and Yuan A, eds), pp 297-324. New York: Springer.

Kimura T, Yamashita S, Fukuda T, Park JM, Murayama M, Mizoroki T, Yoshiike Y, Sahara N, Takashima A (2007) Hyperphosphorylated tau in parahippocampal cortex impairs place learning in aged mice expressing wild-type human tau. EMBO J 26:5143-5152.

Kinoshita E, Kinoshita-Kikuta E, Takiyama K, Koike T (2006) Phosphatebinding tag, a new tool to visualize phosphorylated proteins. Mol Cell Proteomics 5:749-757.

Knowles R, LeClerc N, Kosik KS (1994) Organization of actin and microtubules during process formation in tau-expressing Sf9 cells. Cell Motil Cytoskeleton 28:256-264.

Kumar S, Yin X, Trapp BD, Hoh JH, Paulaitis ME (2002) Relating interactions between neurofilaments to the structure of axonal neurofilament distributions through polymer brush models. Biophys J 82:2360-2372.

Lippens G, Sillen A, Landrieu I, Amniai L, Sibille N, Barbier P, Leroy A, Hanoulle X, Wieruszeski JM (2007) Tau aggregation in Alzheimer's disease: what role for phosphorylation? Prion 1:21-25.

Liu F, Li B, Tung EJ, Grundke-Iqbal I, Iqbal K, Gong CX (2007) Site-specific effects of tau phosphorylation on its microtubule assembly activity and self-aggregation. Eur J Neurosci 26:3429-3436.

Mandelkow EM, Thies E, Trinczek B, Biernat J, Mandelkow E (2004) MARK/PAR1 kinase is a regulator of microtubule-dependent transport in axons. J Cell Biol 167:99-110.

Marx A, Pless J, Mandelkow EM, Mandelkow E (2000) On the rigidity of the cytoskeleton: are MAPs cross-linkers or spacers of microtubules? Cell Mol Biol 46:949-965.

Marx A, Müller J, Mandelkow EM, Hoenger A, Mandelkow E (2006) Interaction of kinesin motors, microtubules, and MAPs. J Muscle Res Cell Motil 27:125-137.

Morel, M, Authelet M, Dedecker R, Brion JP (2010) Glycogen synthase kinase- 3 and the $\mathrm{P} 25$ activator of cyclin dependent kinase 5 increase pausing of mitochondria in neurons. Neuroscience 167:1044-1056.

Morishima-Kawashima M, Hasegawa M, Takio K, Suzuki M, Yoshida H, Titani K, Ihara Y (1995) Proline-directed and non-proline-directed phosphorylation of PHF-tau. J Biol Chem 270:823-829.

Mukhopadhyay R, Hoh JH (2001) AFM force measurements on microtubuleassociated proteins: the projection domain exerts a long-range repulsive force. FEBS Lett 505:374-378.

Planel E, Sun X, Takashima A (2002) Role of GSK-3 $\beta$ in Alzheimer's disease pathology. Drug Dev Res 56:491-510.

Plattner F, Angelo M, Giese KP (2006) The roles of cyclin-dependent kinase 5 and glycogen synthase kinase 3 in tau hyperphosphorylation. J Biol Chem 281:25457-25465.

Rankin CA, Sun Q, Gamblin TC (2005) Pseudo-phosphorylation of tau at Ser202 and Thr205 affects tau filament formation. Mol Brain Res 138:84-93.

Rochlin MW, Wickline KM, Bridgman PC (1996) Microtubule stability decreases axon elongation but not axoplasm production. J Neurosci 16:3236-3246.

Rudrabhatla P, Grant P, Jaffe H, Strong MJ, Pant HC (2010) Quantitative phosphoproteomic analysis of neuronal intermediate filament proteins (NF-M/H) in Alzheimer's disease by iTRAQ. FASEB J 24:4396-4407.

Saito T, Onuki R, Fujita Y, Kusakawa G, Ishiguro K, Bibb JA, Kishimoto T, Hisanaga S (2003) Developmental regulation of the proteolysis of the p35 cyclin-dependent kinase 5 activator by phosphorylation. J Neurosci 23:1189-1197.

Sakaue F, Saito T, Sato Y, Asada A, Ishiguro K, Hasegawa M, Hisanaga S (2005) Phosphorylation of FTDP-17 mutant tau by cyclin-dependent kinase 5 complexed with p35, p25, or p39. J Biol Chem 280:31522-31529.

Seeger MA, Rice SE (2010) Microtubule-associated protein-like binding of the kinesin-1 tail to microtubules. J Biol Chem 285:8155-8162.

Shahpasand K, Ahmadian S, Riazi GH (2008) A possible mechanism for controlling processive transport by microtubule-associated proteins. Neurosci Res 61:347-350.

Shemesh OA, Erez H, Ginzburg I, Spira ME (2008) Tau-induced traffic jams reflect organelles accumulation at points of microtubule polar mismatching. Traffic 9:458-471.

Stamer K, Vogel R, Thies E, Mandelkow E, Mandelkow EM (2002) Tau blocks traffic of organelles, neurofilaments, and APP-vesicles in neurons and enhances oxidative stress. J Cell Biol 156:1051-1063.

Stokin GB, Lillo C, Falzone TL, Brusch RG, Rockenstein E, Mount SL, Raman R, Davies P, Masliah E, Williams DS, Goldstein LS (2005) Axonopathy and transport deficits early in the pathogenesis of Alzheimer's disease. Science 307:1282-1288.

Stoothoff WH, Johnson GV (2005) Tau phosphorylation: physiological and pathological consequences. Biochim Biophys Acta 1739:280-297.

Stoothoff W, Jones PB, Spires-Jones TL, Joyner D, Chhabra E, Bercury K, Fan Z, Xie H, Bacskai B, Edd J, Irimia D, Hyman BT (2009) Differential effect of three-repeat and four-repeat tau on mitochondrial axonal transport. J Neurochem 111:417-427.

Su B, Wang X, Zheng L, Perry G, Smith MA, Zhu X (2010) Abnormal mitochondrial dynamics and neurodegenerative diseases. Biochim Biophys Acta 1802:135-142.

Sudo H, Baas PW (2010) Acetylation of microtubules influences their sensitivity to severing by katanin in neurons and fibroblasts. J Neurosci 30:7215-7226.

Takahashi S, Saito T, Hisanaga S, Pant HC, Kulkarni AB (2003) Tau phosphorylation by cyclin-dependent kinase 5/p39 during brain development reduces its affinity for microtubules. J Biol Chem 278:10506-10515.

Tatebayashi Y, Haque N, Tung YC, Iqbal K, Grundke-Iqbal I (2004) Role of tau phosphorylation by glycogen synthase kinase-3W in the regulation of organelle transport. J Cell Sci 117:1653-1663.

Thies E, Mandelkow EM (2007) Missorting of tau in neurons causes degeneration of synapses that can be rescued by MARK2/Par-1. J Neurosci 27:2896-2907. 
Tokuoka H, Saito T, Yorifuji H, Wei F, Kishimoto T, Hisanaga S (2000) Brain-derived neurotrophic factor-induced phosphorylation of neurofilament-H subunit in primary cultures of embryo rat cortical neurons. J Cell Sci 113:1059-1068.

Trinczek B, Ebneth A, Mandelkow EM, Mandelkow E (1999) Tau regulates the attachment/detachment but not the speed of motors in microtubuledependent transport of single vesicles and organelles. J Cell Sci 112:2355-2367.

Uchida A, Tashiro T, Komiya Y, Yorifuji H, Kishimoto T, Hisanaga S (2004) Morphological and biochemical changes of neurofilaments in aged rat sciatic nerve axons. J Neurochem 88:735-745.

Vershinin M, Carter BC, Razafsky DS, King SJ, Gross SP (2007) Multiple motor based transport and its regulation by Tau. Proc Natl Acad Sci U S A 104:87-92.

Vershinin M, Xu J, Razafsky DS, King SJ, Gross SP (2008) Tuning microtubule-based transport through flamentous MAPs: the problem of dynein. Traffic 9:882-892.

Verwer RW, Sluiter AA, Balesar RA, Baayen JC, Noske DP, Dirven CM,
Wouda J, van Dam AM, Lucassen PJ, Swaab DF (2007) Mature astrocytes in the adult human neocortex express the early neuronal marker doublecortin. Brain 130:3321-3335.

Vossel KA, Zhang K, Brodbeck J, Daub AC, Sharma P, Finkbeiner S, Cui B, Mucke L (2010) Tau reduction prevents Abeta-induced defects in axonal transport. Science 330:198.

Wada Y, Ishiguro K, Itoh TJ, Uchida T, Hotani H, Saito T, Kishimoto T, Hisanaga S (1998) Microtubule-stimulated phosphorylation of tau at Ser202 and Thr205 by cdk 5 decreases its microtubule nucleation activity. J Biochem 124:738-746.

Wang ZF, Li HL, Li XC, Zhang Q, Tian Q, Wang Q, Xu H, Wang JZ (2006) Effects of endogenous beta-amyloid overproduction on tau phosphorylation in cell culture. J Neurochem 98:1167-1175.

Watanabe A, Hasegawa M, Suzuki M, Takio K, Morishima-Kawashima M, Titani K, Arai T, Kosik KS, Ihara Y (1993) In vivo phosphorylation sites in fetal and adult rat tau. J Biol Chem 268:25712-25717.

Yu W, Baas PW (1994) Changes in microtubule number and lenghh during axon differentiation. J Neurosci 14:2818-2829. 